\title{
Shape Preserving Local Histogram Modification
}

\author{
Vicent Caselles * \\ Jose-Luis Lisani * \\ Jean-Michel Morel ${ }^{\dagger}$ \\ Guillermo Sapiro ${ }^{\ddagger}$
}

\begin{abstract}
A novel approach for shape preserving contrast enhancement is presented in this paper. Contrast enhancement is achieved by means of a local histogram equalization algorithm which preserves the level-sets of the image. This basic property is violated by common local schemes, thereby introducing spurious objects and modifying the image information. The scheme is based on equalizing the histogram in all the connected components of the image, which are defined based both on the grey-values and spatial relations between pixels in the image, and following mathematical morphology, constitute the basic objects in the scene. We give examples for both grey-value and color images.
\end{abstract}

Key words: Histogram equalization, local operations, mathematical morphology, levelsets, connected components.

EDICS: 1.3

*Dept. of Mathematics and Informatics, University of Illes Balears, 07071 Palma de Mallorca, Spain, dmivca0@ps.uib.es.

Internal Ácessjen Pate Only of Paris-Dauphine, Place du Marechal de Lattre de Tassigny, 75775 Paris Cedex 16, France, morel@paris9.dauphine.fr.

${ }_{\ddagger}^{\ddagger}$ Hewlett-Packard Labs, 1501 Page Mill Road, Palo Alto, CA 94304, guille@hpl.hp.com. 


\section{Introduction}

Images are captured at low contrast in a number of different scenarios. The main reason for this problem is poor lighting conditions (e.g., pictures taken at night or against the sun rays). As a result, the image is too dark or too bright, and is inappropriate for visual inspection or simple observation. The most common way to improve the contrast of an image is to modify its pixel value distribution, or histogram. A schematic example of the contrast enhancement problem and its solution via histogram modification is given in Figure 1. On the left, we see a low contrast image with two different squares, one inside the other, and its corresponding histogram. We can observe that the image has low contrast, and the different objects can not be identified, since the two regions have almost identical grey values. On the right we see what happens when we modify the histogram in such a way that the grey values corresponding to the two regions are separated. The contrast is improved immediately.
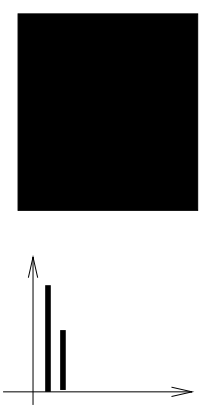
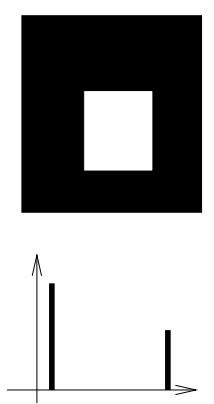

Figure 1: Schematic explanation of the use of histogram modification to improve image contrast.

Histogram modification, and in particular histogram equalization (uniform distributions), is one of the basic and most useful operations in image processing, and its description can be found in any book on image processing. This operation is a particular case of homomorphic transformations: Let $\Omega \subseteq \mathbb{R}^{2}$ be the image domain and $u: \Omega \rightarrow[a, b]$ the given (low contrast) image. Let $h:[a, b] \rightarrow[c, d]$ be a given function which we assume to be increasing. The image $v:=h(u)$ is called an homomorphic transformation of $u$. The particular case of histogram equalization corresponds to selecting $h$ to be the distribution function $H$ of $u$ :

$$
H(\lambda):=\frac{\operatorname{Area}\{x \in \Omega: u(x) \leq \lambda\}}{\operatorname{Area}(\Omega)}
$$

If we assume that $H$ is strictly increasing, then the change of variables

$$
v(x)=(b-a) H(u(x))+a
$$

gives a new image whose distribution function is uniform in the interval $[a, b], a, b \in \mathbb{R}$, $a<b$. This useful and basic operation has an important property which, in spite of being obvious, we would like to acknowledge: Neither it creates or destroys image information. 
As argued by the Mathematical Morphology school [1, 6, 7], the basic operations on images should be invariant with respect to contrast changes, i.e., homomorphic transformations. As a consequence, it follows that the basic information of an image is contained in the family of its binary shadows or level-sets, that is, in the family of sets

$$
X_{\lambda} u:=\{x \in \Omega: u(x) \geq \lambda\},
$$

for all values of $\lambda$ in the range of $u$. Observe that, under fairly general conditions, an image can be reconstructed from its level-sets by the formula $u(x)=\sup \left\{\lambda: x \in X_{\lambda} u\right\}$. If $h$ is a strictly increasing function, the transformation $v=h(u)$ does not modify the family of level-sets of $u$, it only changes its index in the sense that

$$
X_{h(\lambda)} v=X_{\lambda} u \text { for all } \lambda \text {. }
$$

Although one can argue if all operations in image processing must hold this principle, for the purposes of the present paper we shall stick here to this basic principle. There are a number of reasons for this. First of all, a considerable large amount of the research in image processing is based on assuming that regions with (almost) equal grey-values, which are topologicaly connected (see below), belong to the same physical object in the 3D world. Following this, it is natural to assume then that the "shapes" in an given image are represented by its level-sets (we will later see how we deal with noise that produces deviations from the level-sets). Furthermore, this commonly assumed image processing principle will permit to develop a theoretical and practical framework for shape preserving contrast enhancement. This can be extended to other definitions of shape, different from the level-sets morphological approach here assumed. We should note that the level-sets theory is also applicable to a large number of problems beyond image processing [5, 9].

In this paper, we want to design local histogram modification operations which preserve the family of level-sets of the image, that is, following the morphology school, preserve shape. Local contrast enhancement is mainly used to further improve the image contrast and facilitate the visual inspection of the data. As we will see later in this paper, global histogram modification not always produces good contrast, and specially small regions, are hardly visible after such a global operation. On the other hand, local histogram modification improves the contrast of small regions as well, but since the level-sets are not preserved, artificial objects are created. The theory developed in this paper will enjoy the best of both words: The shape-preservation property of global techniques and the contrast improvement quality of local ones.

The recent formalization of multiscale analysis given in [1] leads to a formulation of recursive, causal, local, morphological and geometric invariant filters in terms of solutions of certain partial differential equations of geometric type, providing a new view on many of the basic mathematical morphology operations. One of their basic assumptions was the locality assumption which aimed to translate into a mathematical language the fact that we considered basic operations which were a kind of local average around each pixel or, in other words, only a few pixels around a given sample influence the output value of the operations. Obviously, this excluded the case of algorithms as histogram modification. This is why operations like those in [8] and the one described in this paper are not modeled by these equations, and a novel framework must be developed. 
It is not the goal of this paper to review the extensive research performed in contrast enhancement. We should only note that basically, contrast enhancement techniques are divided in the two groups mentioned above, local and global, and their most popular representatives can be found in any basic book in image processing and computer vision. An early attempt to introduce shape criteria in contrast enhancement was done in [3]. To the best of our knowledge, non of the variations to histogram modification reported in the literature have formally approached the problem of shape preserving contrast enhancement as done in this paper.

\section{Global histogram modification: A variational for- mulation}

We call representatives of $u$ all images of the form $v=h(u)$, where $h$ is a strictly increasing function. The question is which representative of $u$ is the best for our purposes. That will depend, of course, in what our purposes are. We have seen above which is the function $h$ we have to select if we want to normalize the contrast making the distribution function of $u$ uniform. In addition, it was shown in [8] that when equalizing an image $u: \Omega \rightarrow[a, b]$ on the range $[a, b]$ we are minimizing the functional

$$
E(v)=\frac{|\Omega|}{2(b-a)} \int_{\Omega}\left(v(x)-\frac{b-a}{2}\right)^{2} d x-\frac{1}{4} \int_{\Omega} \int_{\Omega}|v(x)-v(z)| d x d z .
$$

The second term of the integral can be understood as a measure of the contrast of the whole image. Thus when minimizing $E(v)$ we are distributing the values of $u$ so that we maximize the contrast. The first term tries to keep the values of $u$ as near as possible to the mean $(b-a) / 2$. When minimizing $E$ on the class of functions with the same family of binary shadows as $u$, we get the equalization of $u$. We will see below how to modify this energy to obtain shape preserving local contrast enhancement.

\section{Connected components}

To be able to extend the global approach to a local setting we have to insist in our main constraint: We have to keep the same topographic map, that is, we have to keep the same family of level-sets of $u$ but we have the freedom to assign them a "convenient" grey level. To make this statement more precise, let us give some definitions (see [10]).

Definition 1 Let $X$ be a topological space. We say that $X$ is connected if it cannot be written as the union of two nonempty closed (open) disjoint sets. A subset $C$ of $X$ is called a connected component if $C$ is a maximal connected subset of $X$, i.e., $C$ is connected and for any connected subset $C_{1}$ of $X$ such that $C \subseteq C_{1}$, then $C_{1}=C$. 
This definition will be applied to subsets $X$ of $\mathbb{R}^{2}$ which are topological spaces with the topology induced from $\mathbb{R}^{2}$, i.e., an open set of $X$ is the intersection of an open set of $\mathbb{R}^{2}$ with $X$. We shall need the following observation which follows from the definition above: Two connected components of a topological space are either disjoint or they coincide; thus the topological space can be considered as the disjoint union of its connected components.

Remark. There are several notions of connectivity for a topological space. One of the most intuitive ones is the notion of arcwise connected (also called connected by arcs). A topological space $X$ is said to be connected by arcs if any two points $x, y$ of $X$ can be joined by an arc, i.e., there exists a continuous function $\gamma:[0,1] \rightarrow X$ such that $\gamma(0)=x, \gamma(1)=y$. In a similar way as above we define the connected components (with respect to this notion of connectivity) as the maximal connected sets. These notions could be used below instead of the one given in Definition 1.

Definition 2 Let $u: \Omega \rightarrow[a, b]$ be a given image. A section of the topographic map of $u$ is a set of the form

$$
X_{\lambda_{1}, \lambda_{2}}=\cup_{\lambda \in\left[\lambda_{1}, \lambda_{2}\right]} C_{\lambda}
$$

where $C_{\lambda}$ is a connected component of $[u=\lambda]$ such that for each $\lambda^{\prime}, \lambda^{\prime \prime} \in\left[\lambda_{1}, \lambda_{2}\right], \lambda^{\prime}<\lambda^{\prime \prime}$, the set

$$
X_{\lambda^{\prime}, \lambda^{\prime \prime}}=\cup_{\lambda \in\left[\lambda^{\prime}, \lambda^{\prime \prime}\right]} C_{\lambda}
$$

is also connected.

Definition 3 Let $u: \Omega \rightarrow[a, b]$ be a given image and let $\left\{X_{\lambda}: \lambda \in[a, b]\right\}$ be the family of its level-sets. We shall say that the mapping $h: \Omega \times \mathbb{R} \rightarrow \mathbb{R}$ is a local contrast change if the following properties hold:

P1: $h$ is continuous in the following sense:

$$
h\left(z, \lambda^{\prime}\right) \rightarrow h(x, \lambda) \quad \text { when } \quad z \rightarrow x, \lambda^{\prime} \rightarrow \lambda, z \in X_{\lambda^{\prime}}, x \in C_{\lambda},
$$

$C_{\lambda}$ being a connected component of $[u=\lambda]$.

P2: $h(x, \cdot)$ is an increasing function of $\lambda$ for all $x \in \Omega$.

P3: $h(x, \lambda)=h(y, \lambda)$ for all $x, y$ are in the same connected component of $[u=\lambda], \lambda \in \mathbb{R}$.

P4: Let $\Gamma$ be a connected set with $u(\Gamma)$ not reduced to a point. Let $v(x)=h(x, u(x))$. Then $v(\Gamma)$ is not reduced to a point.

P5: Let $X_{\lambda_{1}, \lambda_{2}}=\cup_{\left.\lambda_{\in} \in \lambda_{1}, \lambda_{2}\right]} C_{\lambda}$ be a section of the topographic map of $u$ and let $x \in C_{\lambda_{1}}, y \in$ $C_{\lambda_{2}}$. Then $h\left(x, \lambda_{1}\right)<h\left(x, \lambda_{2}\right)$.

Definition 4 Let $u: \Omega \rightarrow[a, b]$ be a given image. We shall say that $v$ is a local representative of $u$ if there exists some local contrast change $h$ such that $v(x)=h(x, u(x)), x \in \Omega$. 
We collect in the next proposition some properties which follow immediately from the definitions above.

Proposition 1 Let $u: \Omega \rightarrow[a, b]$ and let $v(x)=h(x, u(x)), x \in \Omega$, be a local representative of $u$. Then

1. $v(x)=\sup \left\{h(x, \lambda): x \in X_{\lambda} u, x \in \Omega\right\}$. We have that $x \in X_{\lambda} u$ if and only if $x \in$ $X_{h(x, \lambda)} v, x \in \Omega, \lambda \in \mathbb{R}$.

D. $v$ is a continuous function.

3. Let $\Gamma\left(\Gamma^{\prime}\right)$ be a connected component of $[v=\mu]$ (resp. $[u=\lambda]$ ) containing $x, \mu=$ $h(x, \lambda)$. Then $\Gamma=\Gamma^{\prime}$.

4. Let $X_{\lambda_{1}, \lambda_{2}}$ be a section of the topographic map of $u$. Then $X_{\lambda_{1}, \lambda_{2}}$ is also a section of the topographic map of $v$.

\section{Proof.}

1. Is a simple consequence of $\mathbf{P} \mathbf{2}$ in Definition 3 .

2. Is a consequence of $\mathbf{P} \mathbf{1}$ in Definition 3.

3. By P3 of Definition 3, we have $\Gamma^{\prime} \subseteq[v=\mu]$. Since $x \in \Gamma^{\prime}$ and $\Gamma^{\prime}$ is connected, then $\Gamma^{\prime} \subseteq \Gamma$. Now, suppose that

$$
u\left(y_{1}\right)=\inf _{z \in \Gamma} u(z)<\sup _{z \in \Gamma} u(z)=u\left(y_{2}\right), \quad y_{1}, y_{2} \in \Gamma
$$

Thus, $u(\Gamma)$ is not reduced to a point. By $\mathbf{P} 4$ of Definition $3, v(\Gamma)$ is not reduced to a point, a contradiction since $v=\mu$ on $\Gamma$. It follows that $\Gamma \subseteq[u=\lambda]$. Since $\Gamma$ is connected and $x \in \Gamma$, then $\Gamma \subseteq \Gamma^{\prime}$.

4. Let $X_{\lambda_{1}, \lambda_{2}}=\cup_{\lambda \in\left[\lambda_{1}, \lambda_{2}\right]} C_{\lambda}$ be a section of the topographic map of $u$. Let $x_{\lambda} \in C_{\lambda}$ and $\mu_{\lambda}=h\left(x_{\lambda}, \lambda\right)$. By part 3, $C_{\lambda}$ coincides with the connected component of $\left[v=\mu_{\lambda}\right]$ containing $x$ which we denote by $C_{\mu_{\lambda}}(v)$. Let $\mu_{1}=\mu_{\lambda_{1}}, \mu_{2}=\mu_{\lambda_{2}}$. Since, using P5, $\left\{\mu_{\lambda}: \lambda \in\left[\lambda_{1}, \lambda_{2}\right]\right\}=\left[\mu_{1}, \mu_{2}\right]$, then we may write $X_{\lambda_{1}, \lambda_{2}}=\cup_{\mu \in\left[\mu_{1}, \mu_{2}\right]} C_{\mu}(v)$. Now it is easy to see that $X_{\lambda_{1}, \lambda_{2}}$ is a section of the topographic map of $v$.

\section{Remarks.}

1. The previous proposition can be phrased as saying that the set of "objects" contained in $u$ is the same as the set of "objects" contained in $v$, if we understand the "objects" of $u$ as the connected connected components of the level-sets $[\lambda \leq u<\mu], \lambda<\mu$, and respectively for $v$. 
2. Our definition of local representative is contained in the notion of dilation as given in $[6,7]$, Theorem 9.3. Let $\mathcal{U}_{n}$ be a lattice of functions $f: \mathbb{R}^{n} \rightarrow \mathbb{R}^{n}$. A mapping $\Gamma: \mathcal{U}_{n} \rightarrow \mathcal{U}_{n}$ is called a dilation of $\mathcal{U}_{n}$ if and only if it can be written as

$$
\Gamma(f)(x)=\sup \left\{g(x ; y, t): y \in \mathbb{R}^{n}, t \leq f(y)\right\}, \quad x \in \mathbb{R}^{n},
$$

where $g(x ; y, t)$ is a function assigned to each point $(y, t) \in \mathbb{R}^{n} \times \mathbb{R}$ and is possibly different from point to point. Thus, let $h$ be a local contrast change and let $v(x)=$ $h(x, u(x))$. Let us denote by $X_{t}(f, x)$ the connected component of $X_{t} f$ which contains $x$ if $x \in X_{t} f$, otherwise, let $X_{t}(f, x)=\emptyset$. Let $g(x ; y, t):=h(x, t)$ if $X_{t}(f, x) \cap X_{t}(f, y) \neq \emptyset$; and $:=0$ if $X_{t}(f, x) \cap X_{t}(f, y)=\emptyset$. Then $v=\Gamma(u)$.

3. Extending the definition of local contrast change to include more general functions than continuous ones, i.e., to include measurable functions, we can state and prove a converse of Proposition 1, saying that the topographic map contains all the information of the image which is invariant by local contrast changes [2].

\section{Shape preserving contrast enhancement}

We can now state precisely the main question we want to address: what is the best local representative $v$ of $u$, when the goal is to perform local contrast enhancement while preserving the connected components (and level-sets). For that we shall use the energy formulation given in Section 2. Let $A$ be a connected component of the set $[\lambda \leq u<\mu], \lambda, \mu \in \mathbb{R}, \lambda<\mu$. Write

$$
E(v, A):=\frac{|A|}{2(\mu-\lambda)} \int_{A}\left(v(x)-\frac{\mu-\lambda}{2}\right)^{2} d x-\frac{1}{4} \int_{A} \int_{A}|v(x)-v(z)| d x d z .
$$

We then look for a local representative $v$ of $u$ which minimizes $E(v, A)$ for all connected components $A$ of all sets of the form $[\lambda \leq u<\mu], \lambda, \mu \in \mathbb{R}, \lambda<\mu$, or, in other words, the distribution function of $v$ in all connected components of $[\lambda \leq v<\mu]$ is uniform in the range $[\lambda, \mu]$, for all $\lambda, \mu \in \mathbb{R}, \lambda<\mu$. We now show how to solve this problem.

Let us introduce some notation that will make our discussion easier. Without loss of generality we assume that $u: \Omega \rightarrow[0,1]$. Let $\lambda_{k, j}:=j / 2^{k}, k=0,1,2, \ldots, j=0, \ldots, 2^{k}$. We need to assume that $H$, the distribution function of $u$, is continuous and strictly increasing. For that we assume that $u$ is continuous and ${ }^{1}$

$$
\text { Area }\{x \in \Omega: u(x)=\lambda\}=0, \quad \text { for all } \lambda \in \mathbb{R} \text {. }
$$

We shall construct a sequence of functions converging to the solution of the problem. Let $w_{0}=H(u)$ be the histogram equalization of $u$. Suppose that we already constructed $w_{0}, \ldots, w_{i-1}$. Let us construct $w_{i}$. For each $j=0,1, \ldots, 2^{i}-1$, let

$$
O_{i, j}:=\left[\lambda_{i, j} \leq w_{i-1}<\lambda_{i, j+1}\right]
$$

\footnotetext{
${ }^{1}$ This assumption is mainly theoretical and does not necessarily needs to hold for basic practical purposes.
} 
and let $O_{i, j ; r}$ be the connected components of $O_{i, j}, r=1, \ldots, n_{i, j}\left(n_{i, j}\right.$ can be eventually $\left.\infty\right)$. Define

$$
h_{i, j ; r}(\lambda):=\frac{\left|\left[w_{i-1} \leq \lambda\right] \cap O_{i, j ; r}\right|}{\left|O_{i, j ; r}\right|}\left(\lambda_{i, j+1}-\lambda_{i, j}\right)+\lambda_{i, j} .
$$

By our assumption (9), $h_{i, j: r}$ is a continuous strictly increasing function and we can equalize the histogram of $w_{i-1}$ in $O_{i, j ; r}$. Thus, we define

$$
\begin{gathered}
w_{i, j ; r}:=h_{i, j ; r}\left(w_{i-1}\right) \chi_{O_{i, j ; r}}, \\
j=0,1, \ldots, 2^{i}-1, r=1, \ldots, n_{i, j}, \text { and } \\
w_{i}:=\sum_{j=1}^{2^{i}-1} \sum_{r=1}^{n_{i, j}} w_{i, j ; r} \chi_{O_{i, j ; r}} .
\end{gathered}
$$

We will then prove:

Theorem 1 Under the assumption (9) the functions $w_{i}$ have a uniform histogram for all connected components of all "dyadic" sets of the form $\left[\lambda \leq w_{i}<\mu\right]$ where $\lambda, \mu \in\left\{\lambda_{i, j}: j=\right.$ $\left.0, \ldots, 2^{i}\right\}, \lambda<\mu$. Moreover, as $i \rightarrow \infty$, wi converges to a function $w$ which has a uniform histogram for all connected components of all sets $[\lambda \leq w<\mu]$, for all $\lambda, \mu \in[0,1], \lambda<\mu$.

Theorem 2 Let $w$ be the function constructed in Theorem 1. Then $w$ is a local representative of $u$.

The proof of Theorem 1 is based in the next two simple lemmas.

Lemma 1 Let $O_{1}, O_{2} \subseteq \Omega$ such that $O_{1} \cap O_{2}=\emptyset$. Let $u_{i}: O_{i} \rightarrow[a, b)$, $i=1,2$, be two functions with uniform histogram in $[a, b)$. Let $u: O_{1} \cup O_{2} \rightarrow[a, b]$ be given by

$$
u(x)= \begin{cases}u_{1}(x) & \text { if } x \in O_{1} \\ u_{2}(x) & \text { if } x \in O_{2}\end{cases}
$$

Then $u$ has a uniform histogram in $[a, b]$.

Lemma 2 Let $O_{1}, O_{2} \subseteq \Omega$ such that $O_{1} \cap O_{2}=\emptyset$. Let $u_{1}: O_{1} \rightarrow[a, b), u_{2}: O_{2} \rightarrow[b, c)$ be two functions with uniform histogram in $[a, b),[b, c)$, respectively. Assume that

$$
\frac{\left|O_{1}\right|}{\left|O_{1}\right|+\left|O_{2}\right|}=\frac{b-a}{c-a}, \quad \frac{\left|O_{2}\right|}{\left|O_{1}\right|+\left|O_{2}\right|}=\frac{c-b}{c-a} .
$$

Let $u: O_{1} \cup O_{2} \rightarrow[a, c)$ be given by

$$
u(x)= \begin{cases}u_{1}(x) & \text { if } x \in O_{1} \\ u_{2}(x) & \text { if } x \in O_{2}\end{cases}
$$

Then $u$ has a uniform histogram in $[a, c)$. 
Proof of Theorem 1. The first part of the statement follows immediately from the two lemmas above. Now, consider the sequence $\left\{w_{i}\right\}$. Observe that

$$
\left|w_{i}(x)-w_{i+1}(x)\right| \leq \frac{1}{2^{i+1}} \quad \text { for all } x \in \Omega
$$

Indeed, if $w_{i}(x) \in\left[\lambda_{i, j}, \lambda_{i+1,2 j+1}\right)$, then $w_{i+1}(x) \in\left[\lambda_{i+1,2 j}, \lambda_{i+1,2 j+1}\right)$, while, if $w_{i}(x) \in$ $\left[\lambda_{i+1,2 j+1}, \lambda_{i, j+1}\right)$ then $w_{i+1}(x) \in\left[\lambda_{i+1,2 j+1}, \lambda_{i+1,2 j+2}\right)$. The estimate (17) follows. Now, since

$$
w_{N}=\sum_{i=1}^{N}\left(w_{i}-w_{i-1}\right)+w_{0},
$$

and the series on the right hand side is absolutely convergent, then $w_{N}$ converges absolutely and uniformly to some continuous function $w: \Omega \rightarrow[0,1]$. $w$ satisfies the statement above. Indeed, since

$$
\left[w_{i} \leq \lambda_{k, j}\right]=\left[w_{k} \leq \lambda_{k, j}\right] \text { for all } i \geq k, j=0, \ldots, 2^{k}-1
$$

and $w$ is the uniform limit of $w_{N}$, then for all $\epsilon>0$ there is some $N_{0}$ such that

$$
\left[w_{i} \leq \lambda_{k, j}\right] \subseteq\left[w \leq \lambda_{k, j}\right] \subseteq\left[w_{N} \leq \lambda_{k, j}+\epsilon\right]
$$

for all $i \geq k, j=0, \ldots, 2^{k}-1$ and all $N \geq N_{0}$. Letting $\epsilon \rightarrow 0$ it follows that

$$
\left|\left[w \leq \lambda_{k, j}\right]\right|=\lambda_{k, j} \quad \text { for all } k=0,1, \ldots, j=0, \ldots, 2^{k}-1 \text {. }
$$

If $\lambda \in[0,1]$ is not dyadic, let $\lambda_{k, j}, \lambda_{k, j+1}$ be such that $\lambda_{k, j}<\lambda<\lambda_{k, j+1}$. Then

$$
\lambda_{k, j}=\left|\left[w \leq \lambda_{k, j}\right]\right| \leq|[w \leq \lambda]| \leq\left|\left[w \leq \lambda_{k, j+1}\right]\right|=\lambda_{k, j+1} .
$$

Thus by approaching $\lambda$ with dyadic numbers we prove that

$$
|[w \leq \lambda]|=\lambda, \quad \text { for all } \lambda \in[0,1]
$$

Let us mention in passing that the above proof also shows that

$$
\operatorname{Area}([w=\lambda])=0, \quad \text { for all } \lambda \in[0,1] \text {. }
$$

Similarly, one proves that $w$ has a uniform histogram in all connected components of all sets of the form $[\lambda \leq w<\mu]$ for all dyadic numbers $\lambda, \mu \in[0,1], \lambda<\mu$. Now let $\lambda, \mu \in[0,1]$ and let $O$ be a connected component of $[\lambda \leq w<\mu]$. Let $\lambda_{k, j}, \lambda_{k, j^{\prime}}$ be such that $\lambda_{k, j}<\lambda<\mu<\lambda_{k, j^{\prime}}$. Let $O^{\prime}$ be a connected component of $\left[\lambda_{k, j} \leq w<\lambda_{k, j^{\prime}}\right]$ containing $O$. Then

$$
|[w \mid o \leq \eta]| \leq\left|\left[\left.w\right|_{O^{\prime}} \leq \eta\right]\right|=\frac{\eta-\lambda_{k, j}}{\lambda_{k, j^{\prime}}-\lambda_{k, j}}\left|O^{\prime}\right|
$$


for all $\eta \in[\lambda, \mu]$. By property (24), we may approach $\lambda$ and $\mu$ by dyadic numbers while $\left|O^{\prime}\right| \rightarrow|O|$. It follows that

$$
\frac{\left|\left[\left.w\right|_{O} \leq \eta\right]\right|}{|O|} \leq \frac{\eta-\lambda}{\mu-\lambda}
$$

The other inequality is proved in a similar way. It follows that $w$ has a uniform histogram for all connected components of all sets of the form $[\lambda \leq w<\mu]$ for all numbers $\lambda, \mu \in[0,1]$, $\lambda<\mu$.

Proof of Theorem 2. We shall use the notation introduced previously. First we define $h_{0}(x, \lambda)=H(\lambda)(H$ being the global histogram of $u$ ). Let $i \geq 1$. Let $x \in \Omega, \lambda \in[0,1]$. Let $j, r$ be such that $x \in O_{i, j ; r}$. Then we define

$$
h_{i}(x, \lambda)= \begin{cases}\lambda & \text { if } \lambda<\lambda_{i, j}, \\ h_{i, j ; r}(\lambda) & \text { if } \lambda \in\left[\lambda_{i, j}, \lambda_{i, j+1}\right), \\ \lambda & \text { if } \lambda \geq \lambda_{i, j+1} .\end{cases}
$$

It is clear that $h_{0}$ is a local contrast change of $u$. Let us check that $h_{i}$ is a local contrast change of $w_{i-1}, i \geq 1$, i.e., it satisfies P1-P5, for all $i$. To simplify our notation, let us write $W$ instead of $w_{i-1}$.

$\mathbf{P} 1_{i}:$ Let $z \rightarrow x, \lambda^{\prime} \rightarrow \lambda, z \in X_{\lambda^{\prime}}(W), W(x)=\lambda$. Suppose that $\lambda \in\left[\lambda_{i, j}, \lambda_{i, j+1}\right)$ and $x \in O_{i, j ; r}$. Then either $z \in O_{i, j ; r}$ or $z \in O_{i, j-1 ; s}$ for some $s$. If $z \in O_{i, j-1 ; s}$, then $W(z) \in$ $\left[\lambda_{i, j-1}, \lambda_{i, j}\right)$ and $W(z) \rightarrow W(x)$. Hence $W(x)=\lambda_{i, j}$ and $\lambda^{\prime} \rightarrow \lambda_{i, j}, \lambda^{\prime} \in\left[\lambda_{i, j-1}, \lambda_{i, j}\right)$. Then $h_{i}\left(z, \lambda^{\prime}\right)=h_{i, j-1 ; s}\left(\lambda^{\prime}\right) \rightarrow h_{i, j-1 ; s}\left(\lambda_{i, j}\right)=\lambda_{i, j}=h_{i, j ; r}(\lambda)=h_{i}(x, \lambda)$. If $z \in O_{i, j ; r}$, then one easily checks that $h_{i}\left(z, \lambda^{\prime}\right) \rightarrow h_{i}(x, \lambda)$.

$\mathbf{P 2} \mathbf{2}_{i}$ : Follows from the definition of $h_{i}(x, \lambda)$.

$\mathbf{P 3}_{i}$ : Let $x, y$ be in the same connected component of $[W=\lambda], \lambda \in[0,1]$. Let $j$ be such that $\lambda \in\left[\lambda_{i, j}, \lambda_{i, j+1}\right)$. Then $x, y \in O_{i, j ; r}$ for some $r$. Then $h_{i}(x, \lambda)=h_{i}(y, \lambda)=h_{i, j ; r}(\lambda)$.

$\mathbf{P} \mathbf{4}_{i}$ : Let $\Gamma$ be a connected set with $W(\Gamma)$ not reduced to a point. Let $\Gamma_{j ; r}=\Gamma \cap O_{i, j ; r}$. Since $\Gamma=\cup_{j, r} \Gamma_{j ; r}$, for some $j, r, W\left(\Gamma_{j ; r}\right)$ is not reduced to a point. Thus, there exist $x, y \in \Gamma_{j ; r}$ with $W(x)<W(y)$. Then $h_{i}(x, W(x))=h_{i, j ; r}(W(x))<h_{i, j ; r}(W(y))=$ $h_{i}(y, W(y))$. The set $\left\{h_{i}(z, W(z)): z \in \Gamma\right\}$ is not reduced to a point.

P5 $5_{i}$ : Let $X_{\lambda_{1}, \lambda_{2}}=\cup_{\lambda \in\left[\lambda_{1}, \lambda_{2}\right]} C_{\lambda}$ be a section of the topographic map of $W$ and let $x \in$ $C_{\lambda_{1}}, y \in C_{\lambda_{2}}$. Let $j$ be such that $\lambda_{1} \in\left[\lambda_{i, j}, \lambda_{i, j+1}\right), x \in O_{i, j ; r}$. If $\lambda_{2} \in\left[\lambda_{i, j}, \lambda_{i, j+1}\right)$, then $X_{\lambda_{1}, \lambda_{2}} \subseteq O_{i, j ; r}$ since $X_{\lambda_{1}, \lambda_{2}}$ is connected and contains $x$. Thus $y \in O_{i, j ; r}$. Hence $h_{i}\left(x, \lambda_{1}\right)=h_{i, j ; r}\left(\lambda_{1}\right)<h_{i, j ; r}\left(\lambda_{2}\right)=h_{i}\left(x, \lambda_{2}\right)$. Let $k \geq j+1, s$ be such that $\lambda_{2} \in$ $\left[\lambda_{i, k}, \lambda_{i, k+1}\right), y \in O_{i, k ; s}$. Then $h_{i}\left(y, \lambda_{2}\right)=h_{i, k ; s}\left(\lambda_{2}\right) \geq \lambda_{i, k} \geq \lambda_{i, j+1}>h_{i}\left(x, \lambda_{1}\right)$.

Let $H_{0}(x, \lambda)=h_{0}(x, \lambda), H_{i}(x, \lambda)=h_{i}\left(x, H_{i-1}(x, \lambda)\right), i \geq 1$. Observe that $H_{N}(x, u(x))=$ $W_{N}(x)$. Since

$$
H_{N}(x, \lambda)=\sum_{i=0}^{N-1}\left(H_{i}(x, \lambda)-H_{i-1}(x, \lambda)\right)+H_{0}(x, \lambda),
$$


and

$$
\left|H_{i+1}(x, \lambda)-H_{i}(x, \lambda)\right|=\left|h_{i+1}\left(x, H_{i}(x, \lambda)\right)-H_{i}(x, \lambda)\right| \leq \frac{1}{2^{i+1}},
$$

the series in (27) is absolutely and uniformly convergent. Hence $H_{N}(x, \lambda) \rightarrow H(x, \lambda)$ for some function $H(x, \lambda)$. It follows that $H(x, u(x))=w(x)$. Let us now prove that $H(x, \lambda)$ is a local contrast change for $u$.

P1: Let $z \rightarrow x, \lambda^{\prime} \rightarrow \lambda, z \in X_{\lambda^{\prime}}(u), u(x)=\lambda$. Since

$$
H=H_{N}+\sum_{i=N}^{\infty}\left(H_{i+1}-H_{i}\right),
$$

then

$$
\left|H\left(z, \lambda^{\prime}\right)-H(x, \lambda)\right| \leq \frac{2}{2^{N}}+\left|H_{N}\left(z, \lambda^{\prime}\right)-H_{N}(x, \lambda)\right|
$$

Using the corresponding property $\mathbf{H} \mathbf{1}_{i}$ we see that $H\left(z, \lambda^{\prime}\right) \rightarrow H(x, \lambda)$ as $z \rightarrow x$, $\lambda^{\prime} \rightarrow \lambda$.

P2: Follows from $\mathbf{P} 2_{i}$ and the definition of $H$.

P3: Let $x, y$ be in the same connected component of $[u=\lambda], \lambda \in[0,1]$. Then $H_{0}(x, \lambda)=$ $H_{0}(y, \lambda)$ and $x, y$ are in the same connected component of $\left[W_{0}=H_{0}(x, \lambda)\right]$. Then $H_{1}(x, \lambda)=H_{1}(y, \lambda)$. Proceeding iteratively and using $\mathbf{P} \mathbf{3}_{i}$ we get that $H_{N}(x, \lambda)=$ $H_{N}(y, \lambda)$ for all $N$. Letting $N \rightarrow \infty$ we get that $H(x, \lambda)=H(y, \lambda)$.

P4: Let $\Gamma$ be a connected set with $u(\Gamma)$ not reduced to a point. For any $x \in \Gamma$, let $C_{u(x)}(x)$ be the connected component of $[u=u(x)]$ containing $x$. Let $\Gamma^{*}=\cup_{x \in \Gamma} C_{u(x)}(x)$. Since $u(\Gamma)$ not reduced to a point, it contains an interval. This implies that $\operatorname{Area}\left(\Gamma^{*}\right)>0$. Now we observe that $w(\Gamma)=w\left(\Gamma^{*}\right)$. Obviously, $w(\Gamma) \subseteq w\left(\Gamma^{*}\right)$. Now, let $y \in C_{u(x)}(x)$ for some $x \in \Gamma$. Since, by $\mathbf{P} \mathbf{3}, w(x)=w(y)$, we have that $w(y) \in w(\Gamma)$. It follows that $w\left(\Gamma^{*}\right) \subseteq w(\Gamma)$, hence the equality. If $w(\Gamma)$ was reduced to a point $\mu$, then $w\left(\Gamma^{*}\right)=\{\mu\}$. Hence Area $(w=\mu) \geq \operatorname{Area}\left(\Gamma^{*}\right)>0$, contradicting (24). Therefore $w(\Gamma)$ cannot be reduced to a point.

P5: Let $X_{\lambda_{1}, \lambda_{2}}=\cup_{\lambda \in\left[\lambda_{1}, \lambda_{2}\right]} C_{\lambda}$ be a section of the topographic map of $u$ and let $x \in C_{\lambda_{1}}, y \in$ $C_{\lambda_{2}}$. First, using $\mathbf{P} 5_{i}$ and the fact that each $h_{i}$ transforms $X_{\lambda_{1}, \lambda_{2}}$ into a section of the topographic map of $w_{i}$ (Proposition 1), it follows that $H_{N}\left(x, \lambda_{1}\right) \leq H_{N}\left(y, \lambda_{2}\right)$ for all $N$. Letting $N \rightarrow \infty$ we get that $H\left(x, \lambda_{1}\right) \leq H\left(y, \lambda_{2}\right)$. Now, let $z \in X_{\lambda_{1}, \lambda_{2}}$. Since $X_{\lambda_{1}, u(z)}, X_{u(z), \lambda_{2}}$ are also sections of the topographic map of $u$, then by the previous observation we have $H\left(x, \lambda_{1}\right) \leq H(z, u(z)) \leq H\left(y, \lambda_{2}\right)$. If $H\left(x, \lambda_{1}\right)=H\left(y, \lambda_{2}\right)$, then $w(z)=H(z, u(z))=\mu$ for all $z \in X_{\lambda_{1}, \lambda_{2}}$ and some constant $\mu$. Hence Area $(w=\mu) \geq$ Area $\left(X_{\lambda_{1}, \lambda_{2}}\right)>0$, again a contradiction with (24). Thus $H\left(x, \lambda_{1}\right)<H\left(y, \lambda_{2}\right)$. 
Proof of Lemma 1. Let $\lambda \in[a, b]$. Since

$$
[u \leq \lambda]=\left[u_{1} \leq \lambda\right] \cup\left[u_{2} \leq \lambda\right],
$$

it follows that

$$
\begin{aligned}
\frac{|[u \leq \lambda]|}{\left|O_{1}\right|+\left|O_{2}\right|} & =\frac{\left|\left[u_{1} \leq \lambda\right]\right|}{\left|O_{1}\right|} \frac{\left|O_{1}\right|}{\left|O_{1}\right|+\left|O_{2}\right|}+\frac{\left|\left[u_{2} \leq \lambda\right]\right|}{\left|O_{2}\right|} \frac{\left|O_{2}\right|}{\left|O_{1}\right|+\left|O_{2}\right|} \\
& =\frac{\lambda-a}{b-a} \frac{\left|O_{1}\right|}{\left|O_{1}\right|+\left|O_{2}\right|}+\frac{\lambda-a}{b-a} \frac{\left|O_{2}\right|}{\left|O_{1}\right|+\left|O_{2}\right|} \\
& =\frac{\lambda-a}{b-a} .
\end{aligned}
$$

Hence, $u$ has a uniform histogram.

Proof of Lemma 2. Let $\lambda \in\left[a, b\left[\right.\right.$. Since $[u \leq \lambda]=\left[u_{1} \leq \lambda\right]$

$$
\frac{|[u \leq \lambda]|}{\left|O_{1}\right|+\left|O_{2}\right|}=\frac{\left|\left[u_{1} \leq \lambda\right]\right|}{\left|O_{1}\right|} \frac{\left|O_{1}\right|}{\left|O_{1}\right|+\left|O_{2}\right|}=\frac{\lambda-a}{b-a} \frac{b-a}{c-a}=\frac{\lambda-a}{c-a} .
$$

Now, let $\lambda \in[b, c)$. Since $[u \leq \lambda]=O_{1} \cup\left[u_{2} \leq \lambda\right]$,

$$
\begin{aligned}
\frac{|[u \leq \lambda]|}{\left|O_{1}\right|+\left|O_{2}\right|} & =\frac{\left|O_{1}\right|}{\left|O_{1}\right|+\left|O_{2}\right|}+\frac{\left|\left[u_{2} \leq \lambda\right]\right|}{\left|O_{2}\right|} \frac{\left|O_{2}\right|}{\left|O_{1}\right|+\left|O_{2}\right|} \\
& =\frac{b-a}{c-a}+\frac{\lambda-b}{c-b} \frac{c-b}{c-a}=\frac{\lambda-a}{c-a} .
\end{aligned}
$$

We conclude that $u$ has a uniform histogram.

\section{The algorithm and numerical experiments}

The algorithm has been described in the previous section. Let us summarize it here. Let $u: \Omega \rightarrow[0, M]$ be an image whose values have been normalized in $[0, M]$. Let $\lambda_{k, j}:=j M / 2^{k}$, $k=0,1,2, . ., N, j=0, \ldots, 2^{k}$

Step 1: Construct $w_{0}=H(u)$ be the histogram equalization of $u$.

Step 2: Construction of $w_{i}, i=1, \ldots, N$.

Suppose that we already constructed $w_{0}, \ldots, w_{i-1}$. Let us construct $w_{i}$. For each $j=$ $0,1, \ldots, 2^{i}-1$, let

$$
O_{i, j}:=\left[\lambda_{i, j} \leq w_{i-1}<\lambda_{i, j+1}\right],
$$

and let $O_{i, j ; r}$ be the connected components of $O_{i, j}, r=1, \ldots, n_{i, j}$. Let $h_{i, j ; r}$ be the distribution function of $w_{i-1} \chi_{O_{i, j ; r}}$ with values in the range $\left[\lambda_{i, j}, \lambda_{i, j+1}\right]$. 
Then we define

$$
w_{i}:=\sum_{j=1}^{2^{i}-1} \sum_{r=1}^{n_{i, j}} h_{i, j ; r}\left(w_{i-1}\right) \chi_{O_{i, j ; r}} .
$$

Remark. An interesting variant in practice consists in using the mean of $w_{0}$, denoted by $m_{0,1}$, as the value to subdivide the range of $w_{0}$

$$
O_{1,0}=\left[0 \leq w_{0}<m_{0,1}\right], \quad O_{1,1}=\left[m_{0,1} \leq w_{0} \leq M\right] .
$$

Then we equalize $w_{0}$ in all connected components of $O_{1,0}$ in the range [0, $\left.m_{0,1}-1\right]$, respectively in all connected components of $O_{1,1}$ in the range $\left[m_{0,1}, M\right]$. In this way we construct $w_{1}$. Then we compute the mean values of $w_{1}$ in $O_{1,0}, O_{1,1}$. Denote them by $m_{1,1}, m_{1,3}\left(m_{1,2}=m_{0,1}\right)$. Now we use these values to subdivide again $w_{1}$ into four pieces and proceed to equalize the histogram of $w_{1}$ in all connected components of all these pieces. We may continue iteratively in this way until desired.

In Figure 2 we compare the classical local histogram algorithm described in [4] with our algorithm. In the classical algorithm the procedure is to define an $n \times m$ neighborhood and move the center of this area from pixel to pixel. At each location we compute the histogram of the $n \times m$ points in the neighborhood and obtain a histogram equalization (or histogram specification) transformation function. This function is used to map the level of the pixel centered in the neighborhood. The center of the $n \times m$ region is then moved to an adjacent pixel location and the procedure is repeated. In practice one updates the histogram obtained in the previous location with the new data introduced at each motion step. Fig. 2a shows the original image whose level-lines are displayed in Fig. 2b. In Fig. $2 \mathrm{c}$ we show the result of the global histogram equalization of Fig. 2a. Its level-lines are displayed in Fig. 2d. Note how the level-sets lines are preserved, while the contrast of small objects is reduced. Fig. 2e shows the result of the classical local histogram equalization described above $(31 \times 31$ neighborhood $)$, with level-lines displayed in Fig. 2 f. $^{2}$ We see that new level-lines appear thus modifying the topographic map (the set of level-lines) of the original image, introducing new objects. Fig. $2 \mathrm{~g}$ shows the result of our algorithm for local histogram equalization. Its corresponding level-lines are displayed in Fig. $2 \mathrm{~h}$. We see that they coincide with the level-lines of the original image, Fig. $2 b$.

Figure 3 repeats the experiments in Figure 2 for another synthetic image. Fig. 3a has been constructed by cutting half of the right side of Fig. 2a and putting it at the left side of it. Fig. 3b shows the global histogram equalization of Fig. 3a. Fig. 3c shows the result of the classical local histogram equalization described above. Fig. 3d presents the result of our algorithm applied to Fig. 3a. The level-lines off all the figures are given in Fig. 3e-3h respectively. We see how different connected components do not interact in the proposed scheme, and the contrast is improved while preserving the objects in the scene.

Results for a real image are presented in Figure 4. Fig. 4a is the typical "Bureau de l'INRIA image." Fig. 4b is the global histogram equalization of Fig. 4a. Fig. 4c shows an

\footnotetext{
${ }^{2}$ All the level sets for grey-level images are displayed at intervals of 20 grey-values.
} 


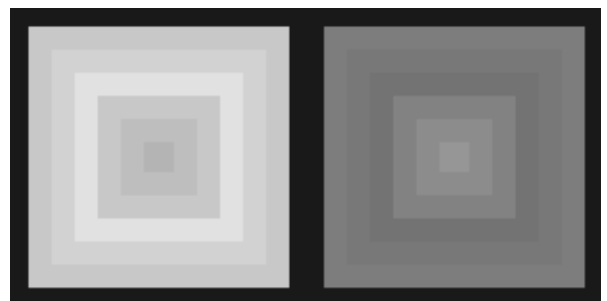

a

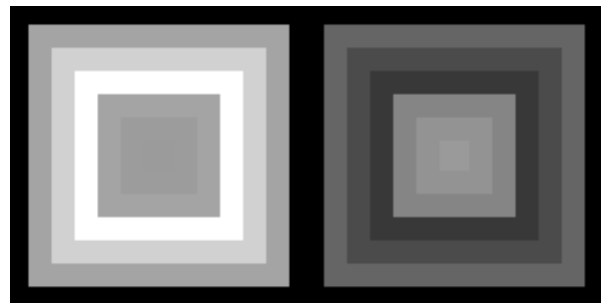

C

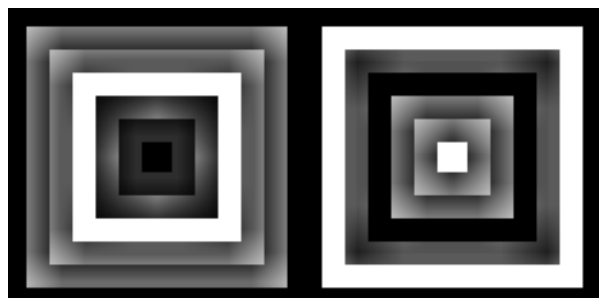

e

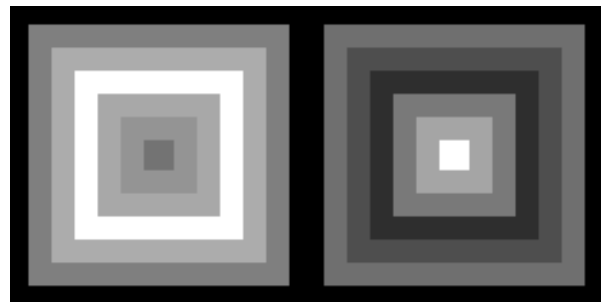

g

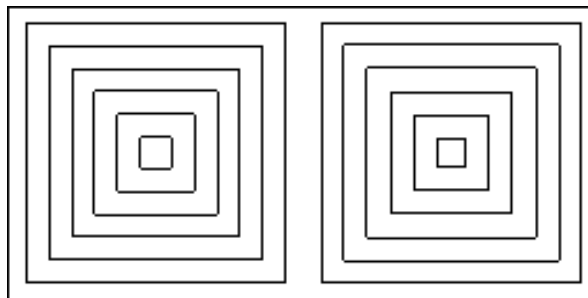

b

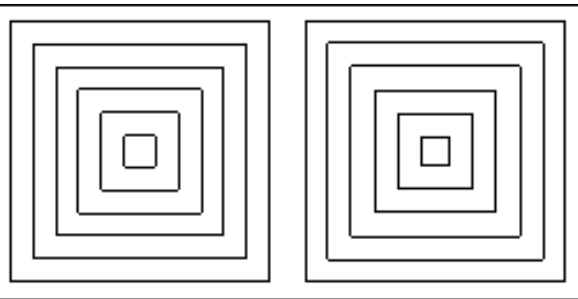

d

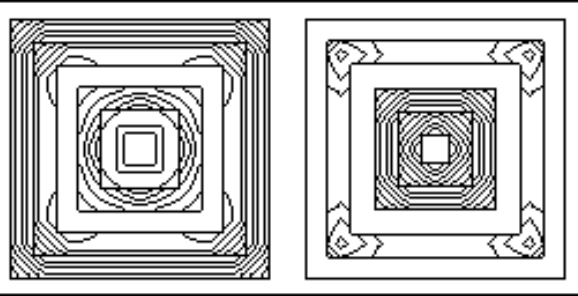

f

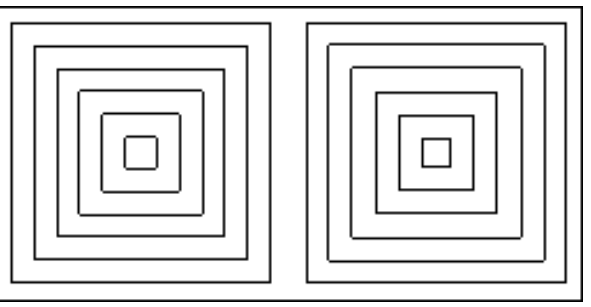

$\mathrm{h}$

Figure 2: Example of the level-sets preservation. The top row shows the original image and its level-sets. The second row shows the result of global histogram modification and the corresponding level-sets. Results of classical local contrast enhancement and its corresponding level-sets are shown in the third row. The last row shows the result of our algorithm. Note how the level-sets are preserved, in contrast with the result on the 3rd row, while the contrast is much better than the global modification. 


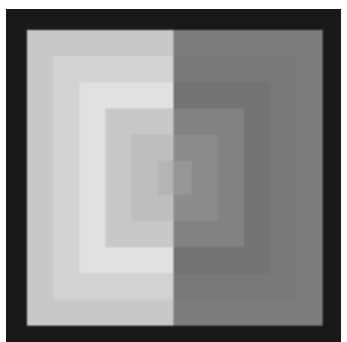

a

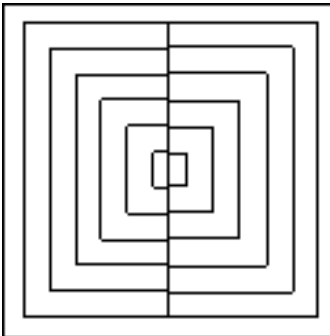

e

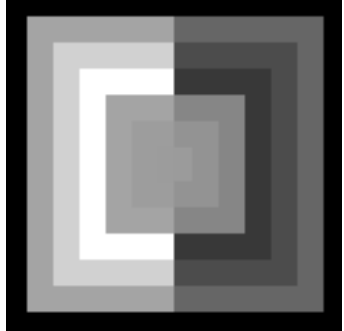

b

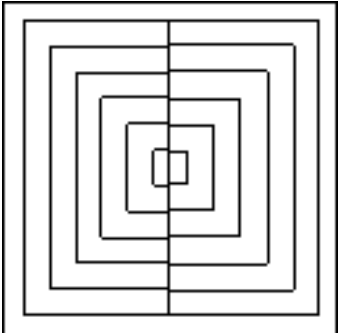

f

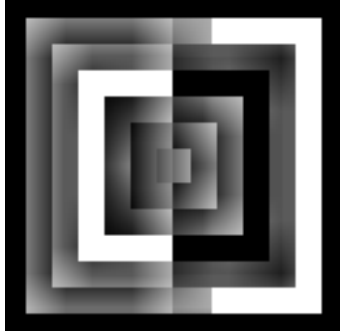

c

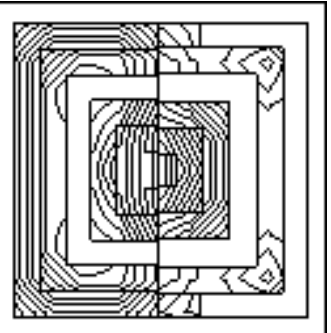

$\mathrm{g}$

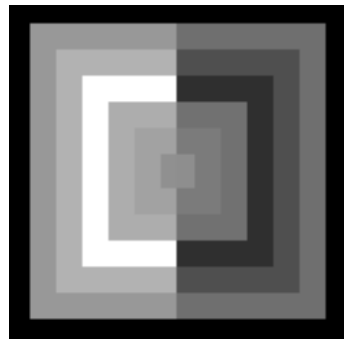

d

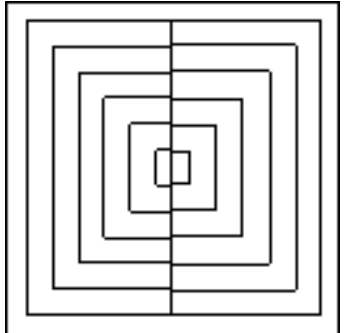

h

Figure 3: Additional example of the level-sets preservation. The first row show the original image, global histogram modification, classical local modification, and the proposed shape preserving local histogram modification. The second row shows the corresponding level-sets.

intermediate step of the proposed algorithm, while Fig. $4 \mathrm{~d}$ is the steady state solution. Note how objects that are not visible in the global modification, like those trough the window, are now visible with the new local scheme.

An additional example is given in Figure 5. Fig. 5a is the original image. Fig. 5b-5d are the results of global histogram equalization, classical local scheme $(61 \times 61$ neighborhood $)$, and our algorithm, respectively.

Experiments with a color image are given in Figure 6, working on the YIQ (luminance and chrominance) color space. In Fig. 6a we present the original image. In Fig. 6b, our algorithm has been applied to the luminance image $\mathrm{Y}$ (maintaining IQ) and then we recomposed the RGB color system. In Fig. 6c, again, we apply the proposed local histogram modification to the color $Y$ channel only, but re-scaling the chrominance vector to maintain the same color point on the Maxwell triangle.

In the last example, Figure 7, we compare the classical local histogram modification scheme with the new one proposed in this paper for a color image, following the same procedure as in Figure 6. Fig. 7a shows the original image, Fig. 7b the one obtained with the classical technique, and Fig. 7c the result of applying our scheme. Note the spurious objects introduced by the classical local scheme. 

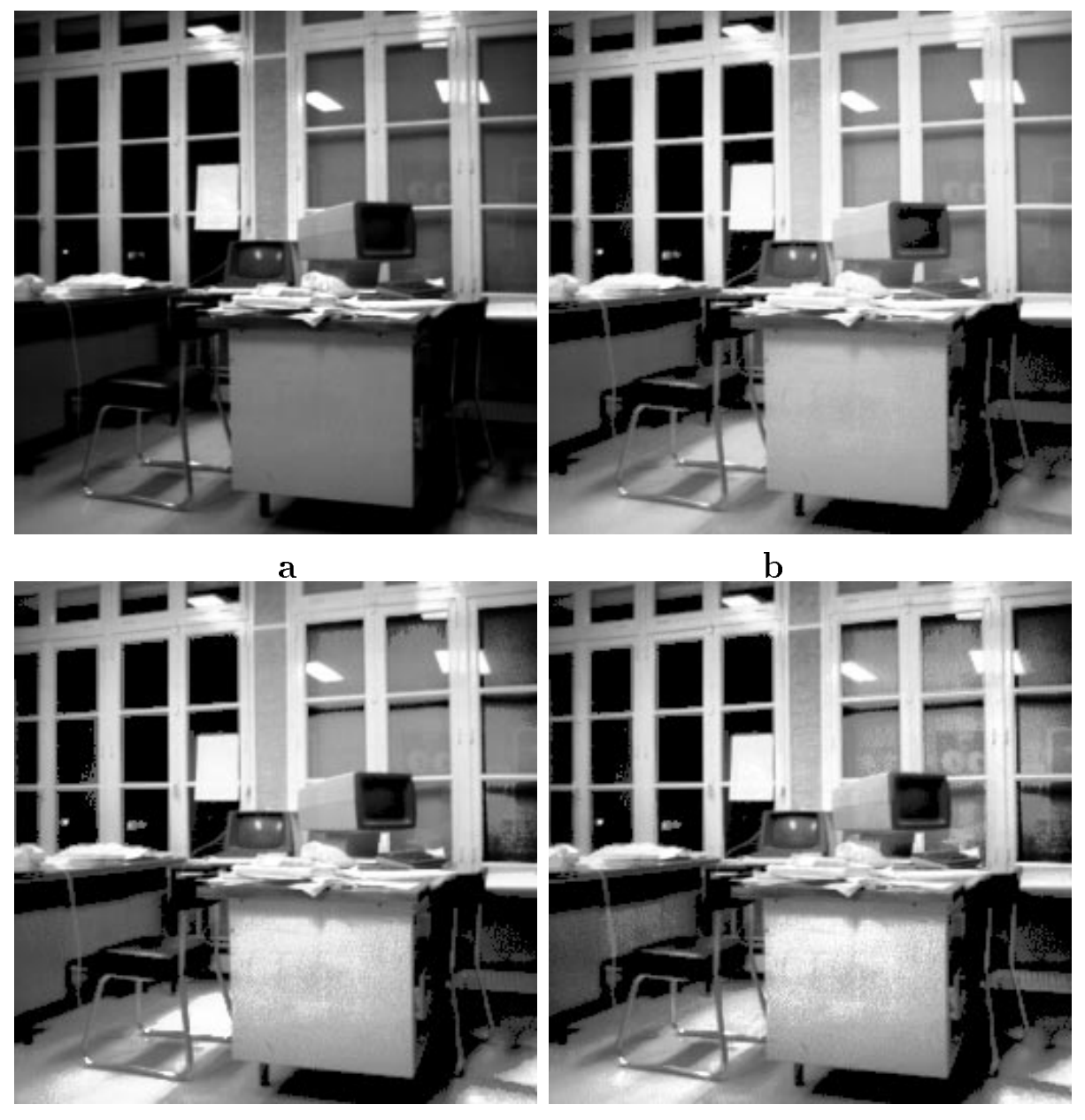

c

d

Figure 4: Example of shape preserving local histogram modification for real data. The first row shows the original image (a) and the result of global histogram modification (b). An intermediate state (c), together with the steady state of the proposed algorithm (d) are shown in the second row. 


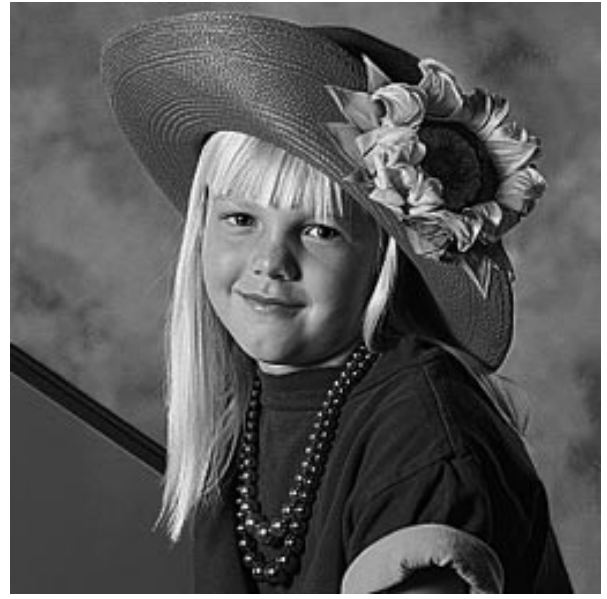

a

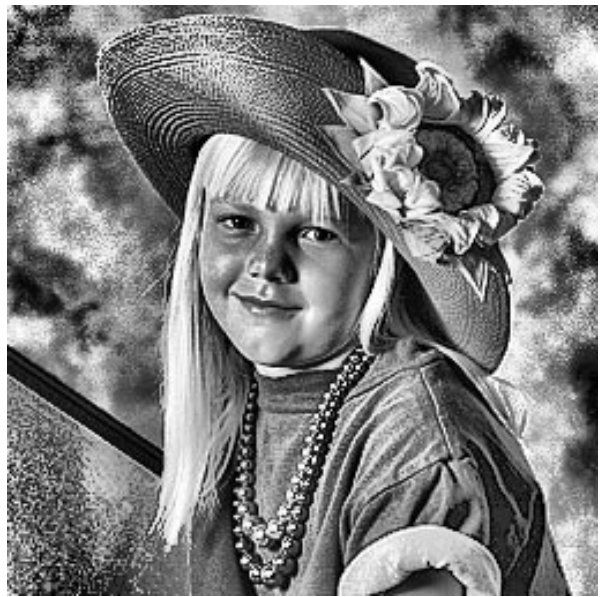

c

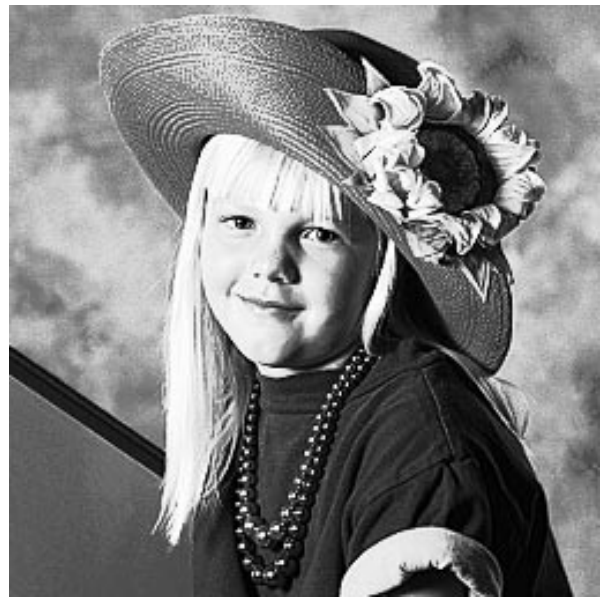

b

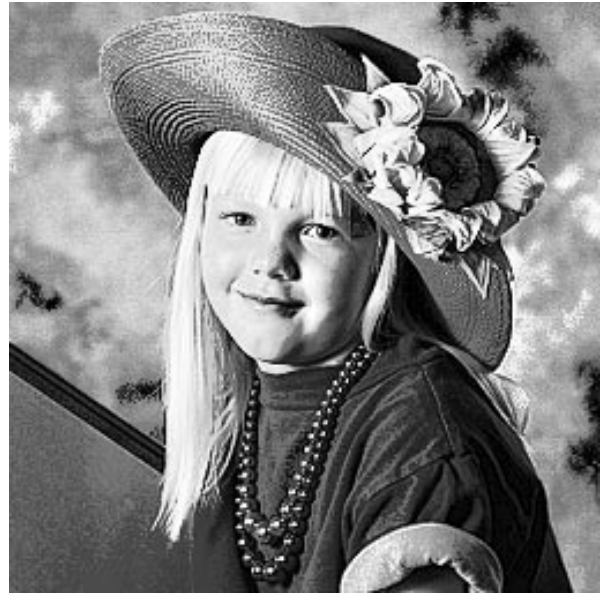

d

Figure 5: Additional example of shape preserving local histogram modification for real data. Fig. $5 a$ is the original image. Fig. 5b-5d are the results of global histogram equalization, classical local scheme $(61 \times 61$ neighborhood), and our algorithm, respectively. 


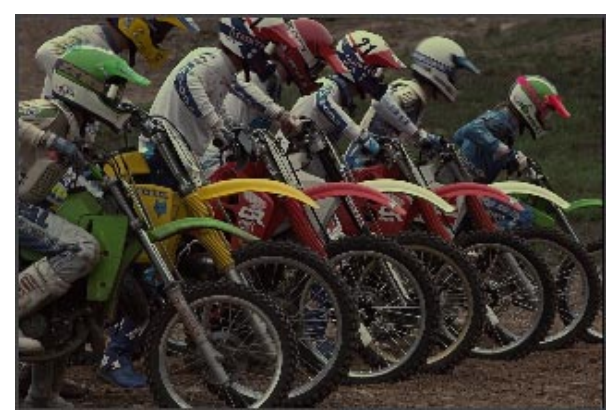

a

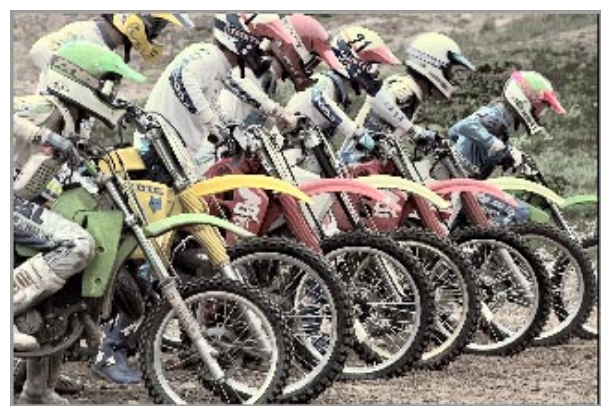

b

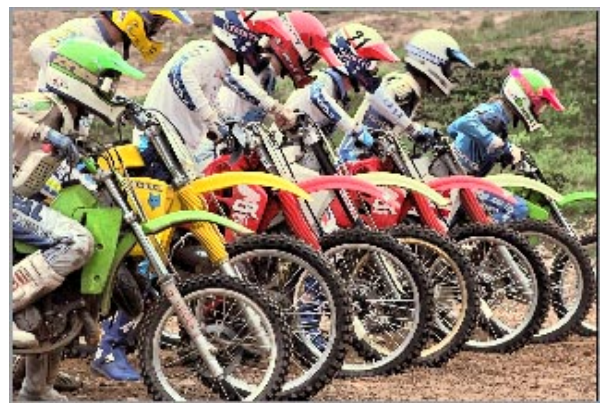

C

Figure 6: Example of local histogram modification of a color image. The original image is shown on the top. The bottom left is the result of applying our algorithm to the $Y$ channel in the YIQ color space. On the right, the algorithm is applied again only to the $Y$ channel, but re-scaling the chrominance vector to maintain the same color point on the Maxwell triangle.

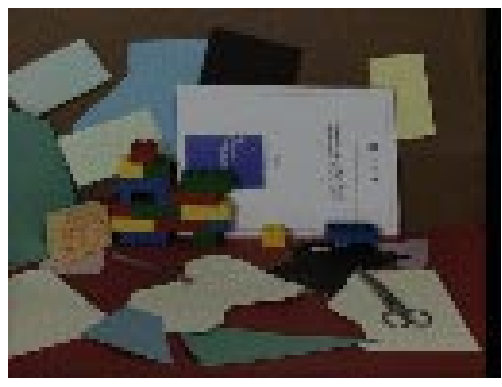

a

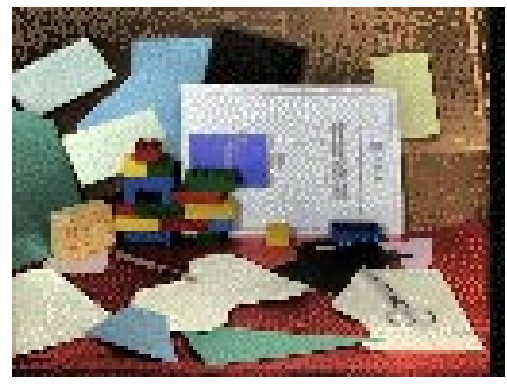

b

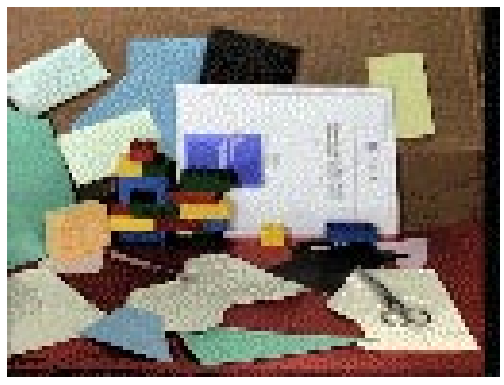

c

Figure 7: Comparison between the classical local histogram modification scheme with the new one proposed in this paper for a color image. Fig. Ta shows the original image, Fig. $7 b$ the one obtained with the classical technique, and Fig. Tc the result of applying our scheme. Note the spurious objects introduced by the classical local scheme. 


\section{Concluding remarks}

This paper presented a novel algorithm for the most basic and (probably) most important operation in image processing: Contrast enhancement. The algorithm is motivated by ideas from the mathematical morphology school, and it holds the main properties of both global and local schemes: It preserves the level-sets of the image, that is, its basic morphological structure, as global histogram modification does, while achieving high contrast results as in local histogram modifications.

A number of problems remain open in this area, and we believe they can be approached with the framework presented in this paper, which complements the results in [8]. One of the open problems is to extend the algorithm to other definitions of connected components, that is, other definitions of objects. In this paper, we define objects as done by the mathematical morphology school, via level-sets, and since this is not the only possible definition, it remains to be shown that a similar approach can be used for other relevant object descriptions. Note that objects can be defined also via optical flow components in video data, or with a concept of connected components in multi-valued images. A general framework for shape preserving contrast enhancement should include these possible definitions as well.

From the energy formulation shown in this paper, equation (5), it is clear that histogram modification is using a measurement of contrast that it is not appropriate at least for human vision. This is because absolute value is not a good model for how humans measure contrast (this value should be at least normalized by the average brightness of the pixel region). The extension of the approach presented in this paper to other models of image contrast is an interesting open area as well. We expect to address these issues elsewhere.

\section{Acknowledgments}

This work was partially supported by DGICYT project, reference PB94-1174, and by the Math, Computer, and Information Sciences Division at ONR. Figure 7 was obtained from the data base of INRIA.

\section{References}

[1] L. Alvarez, F. Guichard, P. L. Lions, and J. M. Morel, "Axioms and fundamental equations of image processing," Arch. Rational Mechanics and Anal. 16:IX, pp. 200$257,1993$.

[2] V. Caselles, J. L. Lisani, J.M. Morel and G. Sapiro, "The information of an image invariant by local contrast changes," pre-print, 1997.

[3] R. Cromartie and S. M. Pizer, "Edge-affected context for adaptive contrast enhancement," Proc. Information Processing in Medical Imaging, Lecture Notes in comp. Science 511, pp. 474-485, Wye, UK, July 1991.

[4] R. C. Gonzalez and P. Wintz, Digital Image Processing, Addison-Wesley, 1987. 
[5] S. J. Osher and J. A. Sethian, "Fronts propagation with curvature dependent speed: Algorithms based on Hamilton-Jacobi formulations," Journal of Computational Physics 79, pp. 12-49, 1988.

[6] J. Serra, Image Analysis and Mathematical Morphology, Academic Press, New York, 1982.

[7] J. Serra Image Analysis and Mathematical Morphology, vol. D: Theoretical Advances., Academic Press, New York, 1988.

[8] G. Sapiro and V. Caselles, "Histogram modification via differential equations," J. of Differential Equations, to appear. A short version appears in Proc. IEEE Int. Conf. Image Proc., Washington-DC, 1995.

[9] J. A. Sethian, Level Set Methods: Evolving Interfaces in Geometry, Fluid Mechanics, Computer Vision and Materials Sciences, Cambridge University Press, Cambridge-UK, 1996.

[10] L. Schwartz, Analyse I. Theorie des Ensembles et Topologie., Hermann, 1991. 